\title{
BMJ Open Independent and combined associations between multiple lifestyle behaviours and academic grades of inner urban and peri-urban high school students: a cross- sectional study in Chongqing, China
}

\author{
Zhengjie Cai (1) , ${ }^{1,2,3,4}$ Jinli Xian (1) , 1,3,4,5 Camila Araujo, ${ }^{6}$ Ziwei Zhang, ${ }^{7}$ \\ Hongyu Zhou, ${ }^{1}$ Huan Peng, ${ }^{1}$ Manoj Sharma, ${ }^{8}$ Yong Zhao (D) $1,3,4,9$
}

To cite: Cai Z, Xian J, Araujo C, et al. Independent and combined associations between multiple lifestyle behaviours and academic grades of inner urban and periurban high school students: a cross-sectional study in Chongqing, China. BMJ Open 2021;11:e049508. doi:10.1136/ bmjopen-2021-049508

- Prepublication history and additional supplemental material for this paper are available online. To view these files, please visit the journal online (http://dx.doi.org/10.1136/ bmjopen-2021-049508)

Received 27 January 2021 Accepted 14 October 2021

Check for updates

(C) Author(s) (or their employer(s)) 2021. Re-use permitted under CC BY-NC. No commercial re-use. See rights and permissions. Published by BMJ.

For numbered affiliations see end of article.

Correspondence to

Dr Yong Zhao;

zhaoyong@cqmu.edu.cn

\section{ABSTRACT}

Objectives This study aims to assess the independent and combined associations between multiple lifestyle behaviours and academic grades of inner urban high school students (IUHSSs) and peri-urban high school students (PUHSSs).

Design A cross-sectional study was conducted. Participants There are 1481 high school students (49.9\% boys) in this study, who were enrolled from one inner urban and two peri-urban schools in Chongqing, China. Outcome measures Academic grades were assessed based on the students' self-reported grade ranking in the last cumulative examination.

Results In IUHSSs and PUHSSs, high frequency of sugarsweetened beverage consumption was unlikely to obtain high academic grades (OR 0.56, 95\% $\mathrm{Cl} 0.32$ to 0.99 and $0.63,95 \% \mathrm{Cl} 0.42$ to 0.96 ), respectively). Among IUHSSs, meeting the recommendations for weekday screen time and egg consumption (OR 1.57, 95\% $\mathrm{Cl} 1.06$ to 2.34 and $1.60,95 \% \mathrm{Cl} 1.04$ to 2.47 , respectively) and high frequency of fruit consumption $(1.67,95 \% \mathrm{Cl} 1.11$ to 2.50) were significantly associated with high academic grades; meeting the recommendation for weekday sleep duration was unlikely to obtain high academic grades (0.46, 95\% Cl 0.21 to 0.98). Among PUHSSs, meeting the recommendations for weekend sleep duration (1.40, $95 \% \mathrm{Cl} 1.02$ to 1.93) and eating dinner regularly (1.55, $95 \% \mathrm{Cl} 1.01$ to 2.37 ) had significant associations with high academic grades. No significant associations were found between physical activity and academic grades in both IUHSSs and PUHSSs ( $p>0.05$ ). Moreover, IUHSSs with 9-13 healthy lifestyle behaviours were 3.25 times more likely to achieve high academic grades than IUHSSs with 1-6 healthy lifestyle behaviours (3.25, $95 \% \mathrm{Cl} 1.96$ to 5.40 ). No significant associations were found in the combined associations between multiple lifestyle behaviours and academic grades among PUHSSs ( $>>0.05)$.

Conclusions Correlations were observed between lifestyle behaviours and academic grades among high school students, and cumulative associations between multiple healthy lifestyle behaviours and academic outcomes appear to be stronger than the independent
Strengths and limitations of this study

- This study is novel as the independent and combined associations between multiple lifestyle behaviours and academic grades among Chinese high school students were assessed.

- Study participants included inner urban and periurban Chinese students, the results in this study could be generalisable to other settings with economic disparities.

- Self-reported data such as lifestyle behaviours and academic grades may result in social desirability bias.

- Unvalidated measures in the questionnaire may raise potential issues related to reliability.

- The cross-sectional nature of this study limits the assessment of causality.

associations. These findings are particularly applicable to IUHSSS.

\section{INTRODUCTION}

High school is a key stage in students' transition to college, academic grades of high school students are closely related to college or university admission, even their future careers and health in adulthood. ${ }^{1}$ A better understanding of modifiable factors affecting high school students' academic grades is important for public health researchers and school principals or management bodies in the high school settings.

Healthy lifestyles, including healthy dietary behaviours, sufficient physical activity and sleep, minimal screen time, etc, are positively associated with students' academic performance. ${ }^{2-4}$ Studies examining the associations between dietary behaviours and academic achievement have typically focused on micronutrient intake, ${ }^{5}$ dietary 
intakes $^{6}$ and breakfast consumption. ${ }^{27}$ Intake of certain micronutrients (ie, iron), high frequency of fruit and vegetable consumption, low consumption of energydense and nutrient-poor food, and regular breakfast consumption all predict the likelihood of good academic grades. ${ }^{5-9}$ Current studies on the association between physical activity and academic grades of children and adolescents are inconsistent. With reported evidence of exercise affecting cognition, ${ }^{10}$ the positive influence of appropriate physical activities on the academic grades of children and adolescents is observed. ${ }^{411}$ However, some studies have reported that additional or enhanced physical exercise do not show positive results in improving academic grades. ${ }^{12}{ }^{13}$ Previous studies investigated the correlation between poor sleep quality and reduced learning capability and poor academic grades among children and adolescents. ${ }^{14}{ }^{15}$ Studies on the correlation between students' screen time and their academic performance have shown that spending considerable time on television, videos or social networking sites appears to be negatively associated with academic grades. ${ }^{16} 17$

Previous studies mainly focused on the association between a single lifestyle factor and students' academic grades. $^{2} 31618$ However, some scholars have proposed that the relationship between healthy lifestyle behaviours and academic performance is not isolated, and the combined associations between multiple healthy lifestyle behaviours and academic performance may be greater than any single behaviour alone. ${ }^{19-21}$ Ickovics et al found that children in grades 5-6 with higher levels of 'health assets' including indicators of a healthy diet, physical activity, screen time and sleep, are two times higher to meet the goal of standardised exams. ${ }^{22}$ Faught et al found that grade 5 students who met the recommendations for 7-9 lifestyle behaviours have higher odds of meeting the expectations for academic achievement compared with those who met three or fewer recommendations. ${ }^{23}$ With the social, cultural and educational context of China, the heavy burden of study for Chinese high school students is common, and maintaining a healthy lifestyle could be ignored. However, having a healthy lifestyle is one of the most important modifiable factors for students' academic grades. Given the importance of academic grades for high school students, the independent and combined associations between lifestyle behaviours and academic grades targeting this population need to be investigated.

Previous studies have demonstrated that there were some factors related to students' academic grades, lifestyle behaviours or both. ${ }^{92-28}$ Faught $e t$ al found that girls had a stronger association with the likelihood of meeting expectations for academic achievement in reading and writing than boys, and the area of residence was associated with students' academic achievement in mathematics. ${ }^{23}$ Some researchers found that students with higher parental education levels were more likely to obtain better academic achievement. ${ }^{9}{ }^{23}$ Li's research showed that boarding school students were more likely to obtain better academic grades than those students who did not board at school. ${ }^{26}$ In addition, Pan and Guan found that students' monthly living expenses were negatively correlated with academic performance, also, whether the student was an only child influenced academic performance. ${ }^{27}$ These factors were also the possible influencing factors of students' lifestyle behaviours. ${ }^{23-25} 28$ Therefore, the above factors were adjusted as potential confounders in this study.

In the context of metropolitan China, 'urban' refers to inner urban and peri-urban areas. Peri-urbanisation refers to the dispersal of urban growth towards the rural surroundings of cities (urban sprawl), thereby creating landscapes that are characterised by urban and rural social and economic activities. ${ }^{29}$ Inner urban districts have higher socioeconomic levels than peri-urban districts in Chongqing, China. ${ }^{30}$ The lifestyle of students differs across various socioeconomic school levels ${ }^{31}$ and is likely to have different influences on the students' academic grades.

To the best of our knowledge, limited studies have investigated the combined associations between lifestyle behaviours and students' academic grades. Hence, this study aims to (1) explore the individual associations between multiple lifestyle behaviours (physical activity before or after school or on weekends, physical activity at school, sleep duration on weekdays and weekends, screen time on weekdays and weekends, consumption frequency of sugar-sweetened beverages (SSBs), breakfast, lunch, dinner, vegetables, fruit, milk and milk alternatives, and the quantity of water and egg consumption) and academic grades among inner urban high school students (IUHSSs) and peri-urban high school students (PUHSSs) in grades 10 and 11 in Chongqing, China, and (2) explore the combined associations between multiple lifestyle behaviours and academic grades in IUHSSs and PUHSSs. This study is expected to effectively promote multiple healthy lifestyle behaviours among high school students from areas with different economic levels and obtain a better understanding of the independent and combined associations between multiple lifestyle behaviours and academic grades of high school students.

\section{METHODS}

\section{Study design and study sample}

A cross-sectional survey was conducted in Chongqing among 1481 high school students. Chongqing is a municipality in midwestern China with 26 districts, including nine inner urban districts and 17 peri-urban districts. One inner urban (Jiangbei) and two peri-urban districts (Dazu and Kaizhou) were randomly chosen in Chongqing, and one high school was randomly selected from each designated district. A total of 6-12 classes were randomly selected from each school, and all students in the selected classes were invited to participate in this study. High school students in grades 10 and 11 were eligible to participate in this study. Students who have a history of major diseases, chronic health conditions or 
mental trauma were excluded in this study. Investigators explained the research and distributed the questionnaire to all students, and students promptly completed the anonymous questionnaire independently.

According to the research of Yan et $a l^{32}$, nearly $48 \%$ of middle-school and high-school students reported having good grade rankings in their final examination. According to the sample size calculation formula of the cross-sectional study $N=\left(Z_{\alpha}^{2} \times p \times q\right) / d^{2}$, we set $p=0.48$, $q=1-\mathrm{p}=0.52$, and margin of error $\mathrm{d}=0.10 \times \mathrm{p}=0.048, \mathrm{Z}_{\alpha}$ $=1.96$, the calculated sample size was 416. In the survey, the actual total sample size included 1481 individuals.

\section{Patient and public involvement}

Patients or the public were not involved in the design, or conduct, or reporting, or dissemination plans of our research.

\section{Exposure}

The descriptions of 15 questions in terms of lifestyle behaviours (physical activity, sleep duration, screen time and dietary behaviours) are presented in online supplemental table 1 . Healthy lifestyle behaviours were assessed based on the recommended guidelines or previous studies. High school students who engage in moderateto-high-intensity physical activity for more than 1 hour a day and whose screen time is less than 2 hours a day meet the physical activity guidelines for Chinese children and adolescents. ${ }^{33}$ Physical activity was categorised into $\geq 1$ hour and $<1$ hour, and daily screen time was categorised into $\geq 2$ hours and $<2$ hours. Insufficient sleep was defined as less than 8 hours a day for high school students, as recommended by the Chinese Dietary Guideline $2016 .{ }^{34}$ Sleep duration was categorised into $\geq 8$ hours and $<8$ hours. In terms of dietary behaviours, participants were asked about the consumption frequency of SSBs, breakfast, lunch, dinner, vegetables, fruit, milk and milk alternatives, and the quantity of water and egg consumption. As recommended by the Chinese Dietary Guideline $2016,{ }^{34}$ water and egg consumption were divided into two categories, namely, meeting the guideline and not meeting the guideline. Consumption of SSBs less than once a week, consumption of breakfast, lunch and dinner five or more times per week, and consumption of vegetables, fruit, and milk and milk alternatives more than once per day were considered as healthy dietary behaviours. $^{35-37}$

\section{Outcome}

Academic grades were approximately assessed based on the students' self-reported ranking in the last cumulative examination in their grades, with the options being the top 25\%, 25\%-50\%, 50\%-75\% and lowest 25\%. With reference to previous studies, ${ }^{42}$ academic grades were dichotomised into good (top 50\%) and poor (last 50\%) for statistical analyses.

\section{Potential confounders}

Analyses were adjusted in terms of school areas, age, gender (male/female), residence (urban/rural), being an only child in the family (yes/no), boarding school (yes/ no), average monthly living expenses (low: $\leq$ RMB800, medium: RMB801-RMB1200 and high: >RMB1200), and parental education level (low: primary school or below, medium: secondary school/secondary vocational school/ high school and high: college or above).

\section{Statistical analyses}

All analyses were performed with the Statistical Package for Social Sciences (SPSS) software V.21 (IBM). Invalid or missing data were excluded, and all data were doublechecked. The categorical variables were described using frequency and percentiles, and owing to the non-normal distribution of age, we described age using median and IQR. $\chi^{2}$ tests were conducted to examine the differences in lifestyle behaviours between IUHSSs and PUHSSs. The independent and combined associations between multiple lifestyle behaviours and academic grades were assessed through multivariate logistic regressions. ORs and their corresponding 95\% CIs were reported. An unadjusted logistic regression was used to assess the independent associations between multiple lifestyle behaviours and academic grades in all students, IUHSSs and PUHSSs, respectively. In all students, the adjusted model reported the results of multivariate regression analysis after adjusting the potential confounders (school area, age, gender, residence, being an only child, boarding at the school, average monthly living expenses, and parental education level) and all lifestyle behaviours. In IUHSSs and PUHSSs, the adjusted model reported the results of multivariate regression analysis after adjusting age, gender, residence, being an only child, boarding at the school, average monthly living expenses, and parental education level and all lifestyle behaviours, respectively. The effect of the number of healthy lifestyle behaviours was considered to assess the combined associations between multiple lifestyle behaviours and academic grades. The total number of healthy lifestyle behaviours ranged from 1 to 13, and three categories of healthy lifestyle behaviours were constructed in accordance with the frequency distribution of the number of healthy lifestyle behaviours: low (1-6 healthy lifestyle behaviours, $40.0 \%$ ), medium (7-8 healthy lifestyle behaviours, $38.8 \%$ ) and high (9-13 healthy lifestyle behaviours, 21.2\%). Treating the number of healthy lifestyle behaviours as both categorical and continuous variables, univariable and multivariable regression models were used to assess the cumulative associations between multiple lifestyle behaviours and academic grades. A $\mathrm{p}<0.05$ (two-sided) was considered as statistical significance.

\section{RESULTS}

\section{Sample characteristics}

Table 1 shows the sample characteristics of participants. IUHSSs comprised $45.8 \%$ out of 1481 high school 


\begin{tabular}{|c|c|c|}
\hline Variables & & $\begin{array}{l}\text { n (\%) or } \\
\text { mean } \pm \text { SD }\end{array}$ \\
\hline \multirow[t]{2}{*}{ School areas } & Inner urban & $678(45.8)$ \\
\hline & Peri-urban & $803(54.2)$ \\
\hline Age (median (IQR)) & & $16.0(1.0)$ \\
\hline \multirow[t]{2}{*}{ Gender } & Boy & 739 (49.9) \\
\hline & Girl & $742(50.1)$ \\
\hline \multirow[t]{2}{*}{ Residence } & City & $957(64.6)$ \\
\hline & Village & $524(35.4)$ \\
\hline \multirow[t]{2}{*}{ Only-child status } & Yes & $525(35.5)$ \\
\hline & No & $956(64.5)$ \\
\hline \multirow[t]{2}{*}{ Boarding school } & Yes & $859(58.0)$ \\
\hline & No & $622(42.0)$ \\
\hline \multirow{3}{*}{$\begin{array}{l}\text { Average monthly living } \\
\text { expenses (RMB) }\end{array}$} & Low & $861(58.1)$ \\
\hline & Medium & 465 (31.4) \\
\hline & High & $155(10.5)$ \\
\hline \multirow{3}{*}{$\begin{array}{l}\text { Father's educational } \\
\text { level }\end{array}$} & Low & $163(11.0)$ \\
\hline & Medium & $832(56.2)$ \\
\hline & High & $486(32.8)$ \\
\hline \multirow{3}{*}{$\begin{array}{l}\text { Mother's educational } \\
\text { level }\end{array}$} & Low & $278(18.8)$ \\
\hline & Medium & $808(54.6)$ \\
\hline & High & $395(26.6)$ \\
\hline \multirow[t]{2}{*}{ Academic grades } & Top 50\% & $945(63.8)$ \\
\hline & Last $50 \%$ & $536(36.2)$ \\
\hline
\end{tabular}

students (49.9\% boys). The median age is 16.0. Among the students, $64.6 \%$ lived in the city, $64.5 \%$ had siblings and $58.0 \%$ were boarding school. More than half (58.1\%) of the students' average monthly living expenses were less than RMB800. More than half of the parental education level were medium (56.2\% and $54.6 \%$, respectively). And $63.8 \%$ of the students' academic grades were ranked as top $50 \%$.

\section{Lifestyle behaviours of participants}

Table 2 shows the comparison of lifestyle behaviours between IUHSSs and PUHSSs. The percentages of students meeting the recommendations of lifestyle behaviours were: $5.6 \%$ and $19.5 \%$ for daily physical activity before or after school or on weekends and at school, respectively; $10.8 \%$ and $70.5 \%$ for daily sleep duration on weekdays and on weekends, respectively; $81.4 \%$ and $18.4 \%$ for daily screen time on weekdays and on weekends, respectively; and $18.5 \%$ and $23.0 \%$ for water and egg consumption, respectively. And $27.3 \%$ of the students drank SSBs less than once a week. Breakfast, lunch and dinner consumption five times or more per week were $83.8 \%, 96.2 \%$ and $79.1 \%$, respectively. Vegetable, fruit, and milk and milk alternatives consumption more than once per day were $79.1 \%, 42.4 \%$ and $42.7 \%$, respectively.
All lifestyle behaviours varied between IUHSSs and PUHSSs except for physical activity at school, frequency of breakfast, dinner and vegetable intake, and water and egg consumption $(\mathrm{p}<0.05)$.

\section{Logistic regression for identifying the independent and} combined associations of multiple lifestyle behaviours and academic grades among IUHSSs and PUHSSs

The results of the logistic regression analysis for identifying the independent associations between multiple lifestyle behaviours and academic grades are shown in table 3 .

In the adjusted model, among all students, students who met the recommendations for sleep duration on weekends (OR 1.39, 95\% CI 1.10 to 1.77 ) and eating dinner regularly ( $\geq 5$ times/week vs $<5$ times/week: OR $1.47,95 \%$ CI 1.11 to 1.95 ) were significantly correlated with high academic grades; students with high frequency of SSBs consumption ( $\geq 5$ times/week vs <once/week: OR $0.66,95 \%$ CI 0.48 to 0.92 ) were unlikely to obtain high academic grades.

In the adjusted model, among IUHSSs, meeting the recommendations for screen time on weekdays (OR 1.57, $95 \%$ CI 1.06 to 2.34 ) and daily egg consumption (OR $1.60,95 \%$ CI 1.04 to 2.47 ) and high frequency of fruit consumption ( $\geq$ once/week vs <once/week: OR 1.67, 95\% CI 1.11 to 2.50 ) were still significantly correlated with high academic grades; students who met the recommendations for sleep duration on weekdays (OR $0.46,95 \%$ CI 0.21 to 0.98 ) and high frequency of SSBs consumption ( $\geq 5$ times/week vs <once/week: OR $0.56,95 \%$ CI 0.32 to 0.99 ) were unlikely to obtain high academic grades. Among PUHSSs, meeting the recommendations for sleep duration on weekends (OR 1.40, 95\% CI 1.02 to 1.93 ) and eating dinner regularly ( $\geq 5$ times/week vs $<5$ times/week: OR $1.55,95 \%$ CI 1.01 to 2.37 ) were significantly correlated with high academic grades; students with high frequency of SSBs consumption (3-4 times/week vs <once/week: OR $0.63,95 \%$ CI 0.42 to 0.96 ) were unlikely to obtain high academic grades.

The results of the logistic regression analysis for identifying the combined associations between multiple lifestyle behaviours and academic grades are shown in table 4 . In the adjusted model, the number of healthy lifestyle behaviours in all students was positively associated with academic grades (OR 1.12, 95\% CI 1.05 to 1.19), and students who had 9-13 healthy lifestyle behaviours were more likely to obtain high academic grades than those who had 1-6 healthy lifestyle behaviours (OR 1.74, $95 \%$ CI 1.28 to 2.37 ).

In the adjusted model, the number of healthy lifestyle behaviours among IUHSSs was positively associated with academic grades (OR 1.23, 95\% CI 1.11 to 1.35). And IUHSSs who had 7-8 healthy lifestyle behaviours were more likely to obtain high academic grades than those who had 1-6 healthy lifestyle behaviours (OR 1.54, 95\% CI 1.06 to 2.24), IUHSSs who had 9-13 healthy lifestyle behaviours had 3.25 times higher odds of obtaining high 
Table 2 The comparison of lifestyles behaviours of students between inner urban and peri-urban high schools in Chongqing, China $(n=1481)$

\begin{tabular}{|c|c|c|c|c|c|}
\hline Variables & Total population & Inner urban $(n=678)$ & Peri-urban $(n=803)$ & $\chi^{2}$ & $P$ value \\
\hline \multicolumn{6}{|c|}{ Physical activity before or after school or on weekends } \\
\hline$<1$ hour & $1398(94.4)$ & $631(93.1)$ & 767 (95.5) & 4.17 & 0.04 \\
\hline$\geq 1$ hour & $83(5.6)$ & $47(6.9)$ & $36(4.5)$ & & \\
\hline \multicolumn{6}{|c|}{ Physical activity at school } \\
\hline$<1$ hour & $1192(80.5)$ & $547(80.7)$ & $645(80.3)$ & 0.03 & 0.86 \\
\hline$\geq 1$ hour & $289(19.5)$ & $131(19.3)$ & $158(19.7)$ & & \\
\hline \multicolumn{6}{|c|}{ Sleep time on weekdays } \\
\hline$<8$ hours & $1321(89.2)$ & $643(94.8)$ & $678(84.4)$ & 41.30 & $<0.001$ \\
\hline$\geq 8$ hours & $160(10.8)$ & $35(5.2)$ & $125(15.6)$ & & \\
\hline \multicolumn{6}{|c|}{ Sleep time on weekends } \\
\hline$<8$ hours & $437(29.5)$ & $171(25.2)$ & $266(33.1)$ & 11.04 & $<0.001$ \\
\hline$\geq 8$ hours & $1044(70.5)$ & $507(74.8)$ & $537(66.9)$ & & \\
\hline \multicolumn{6}{|c|}{ Screen time on weekdays } \\
\hline$\geq 2$ hours & $276(18.6)$ & $157(23.2)$ & $119(14.8)$ & 16.85 & $<0.001$ \\
\hline$<2$ hours & $1205(81.4)$ & $521(76.8)$ & $684(85.2)$ & & \\
\hline \multicolumn{6}{|c|}{ Screen time on weekends } \\
\hline$\geq 2$ hours & 1208 (81.6) & $581(85.7)$ & $627(78.1)$ & 14.16 & $<0.001$ \\
\hline$<2$ hours & $273(18.4)$ & $97(14.3)$ & $176(21.9)$ & & \\
\hline \multicolumn{6}{|c|}{ Sugar-sweetened beverages } \\
\hline$\geq 5$ times/week & $343(23.2)$ & $174(25.7)$ & $169(21.1)$ & 51.30 & $<0.001$ \\
\hline 3-4 times/week & $333(22.5)$ & $169(24.9)$ & $164(20.4)$ & & \\
\hline 1-2 times/week & $401(27.1)$ & $211(31.1)$ & $190(23.6)$ & & \\
\hline$<$ Once/week & $404(27.3)$ & $124(18.3)$ & $280(34.9)$ & & \\
\hline \multicolumn{6}{|l|}{ Breakfast } \\
\hline$<5$ times/week & $240(16.2)$ & $110(16.2)$ & $130(16.2)$ & 0.00 & 0.99 \\
\hline$\geq 5$ times/week & $1241(83.8)$ & $568(83.8)$ & $673(83.8)$ & & \\
\hline \multicolumn{6}{|l|}{ Lunch } \\
\hline$<5$ times/week & $56(3.8)$ & $14(2.1)$ & $42(5.2)$ & 10.12 & 0.001 \\
\hline$\geq 5$ times/week & 1425 (96.2) & $664(97.9)$ & $761(94.8)$ & & \\
\hline \multicolumn{6}{|l|}{ Dinner } \\
\hline$<5$ times/week & 309 (20.9) & $155(22.9)$ & $154(19.2)$ & 3.02 & 0.08 \\
\hline$\geq 5$ times/week & $1172(79.1)$ & $523(77.1)$ & $649(80.8)$ & & \\
\hline \multicolumn{6}{|l|}{ Vegetables } \\
\hline$<$ Once/day & $310(20.9)$ & $156(23.0)$ & $154(19.2)$ & 3.26 & 0.07 \\
\hline$\geq$ Once/day & $1171(79.1)$ & $522(77.0)$ & $649(80.8)$ & & \\
\hline \multicolumn{6}{|l|}{ Fruit } \\
\hline$<$ Once/day & $853(57.6)$ & $349(51.5)$ & $504(62.8)$ & 19.18 & $<0.001$ \\
\hline$\geq$ Once/day & $628(42.4)$ & $329(48.5)$ & $299(37.2)$ & & \\
\hline \multicolumn{6}{|c|}{ Milk and milk alternatives } \\
\hline$<$ Once/day & $849(57.3)$ & $290(42.8)$ & $559(69.6)$ & 108.26 & $<0.001$ \\
\hline$\geq$ Once/day & $632(42.7)$ & $388(57.2)$ & $244(30.4)$ & & \\
\hline \multicolumn{6}{|c|}{ Meeting water intake recommendation } \\
\hline No & $1207(81.5)$ & $544(80.2)$ & $663(82.6)$ & 1.32 & 0.25 \\
\hline Yes & $274(18.5)$ & $134(19.8)$ & $140(17.4)$ & & \\
\hline
\end{tabular}


Table 2 Continued

\begin{tabular}{|c|c|c|c|c|c|}
\hline Variables & Total population & Inner urban $(n=678)$ & Peri-urban $(n=803)$ & $\chi^{2}$ & $P$ value \\
\hline No & $1141(77.0)$ & 508 (74.9) & $633(78.8)$ & 3.17 & 0.08 \\
\hline
\end{tabular}

academic grades than those who had 1-6 healthy lifestyle behaviours (OR $3.25,95 \%$ CI 1.96 to 5.40 ). No combined association was found between lifestyle behaviours and academic grades among PUHSSs $(\mathrm{p}>0.05)$.

\section{DISCUSSION}

Lifestyle behaviours of high school students appear to be associated with academic grades, and the cumulative associations between multiple healthy lifestyle behaviours and their academic outcomes seem to be stronger than the independent associations. These findings are particularly applicable to IUHSSs. Lifestyle behaviours are closely related to education, the findings in this study revealed that multiple healthy lifestyle behaviours of high school students may be positively associated with their academic achievement.

Previous studies reported that meeting the recommendation of sleep duration was positively associated with the academic grades of children and adolescents. ${ }^{14} 15$ Sleep is thought to play a crucial and specific role in memory consolidation, and lack of sleep was linked to increased fatigue and sleepiness, and poor attention and cognition. ${ }^{38}$ However, the association between sleep duration and academic grades among IUHSSs and PUHSSs was inconsistent in this study. Among PUHSSs, meeting the recommendation for sleep duration on weekends had a positive association with students' academic grades. By contrast, a negative association was found between adhering to the recommended sleep duration on weekdays and academic grades among IUHSSs. Education development is usually synchronized with economic development, and regional differences in education development in Chongqing were found. ${ }^{40}$ Inner urban high schools may have stricter rules and tighter schedules compared with peri-urban high schools, and insufficient sleep may be common among IUHSSs. The result of this study showed that extremely few IUHSSs (5.2\%) had 8 hours or more sleep duration on weekdays. We speculated that IUHSSs who slept 8 hours or more on weekdays may sleep at other times, such as during class or independent study time, leading to less time spent studying. In addition, IUHSSs with good academic grades may spend more time in learning than those with poor academic grades, which could be another possible explanation for the negative association between insufficient sleep time on weekdays and good academic grades among IUHSSs.

A previous systematic review and meta-analysis indicated that television viewing and video game playing were negatively associated with academic outcomes, while overall screen time (including watching television, playing electronic games, receiving electronic news and study materials, and using social networking sites) was not associated with academic outcomes among children and adolescents aged 4-18 years. ${ }^{16}$ The screen time surveyed in this study was overall screen time. The results revealed that meeting the recommendation for screen time on weekdays had a positive association with academic grades, and the association between weekend screen time and academic performance was statistically insignificant among IUHSSs. One possible explanation could be that Chinese high school students are not allowed to use electronic devices on weekdays owing to the strict regulations, but they may use electronic devices in 'cram schools' on weekends for receiving electronic news and study materials that may neutralise the negative association between screen-based behaviours and academic grades. No association between screen time and academic grades was found among PUHSSs. The reason could be that students from inner urban areas may have greater access to screen devices and would be more likely to engage in screenbased behaviours compared with those from peri-urban high schools.

The results in this study showed increased odds of high academic grades among PUHSSs who had regular dinner consumption, and no association was observed between breakfast, lunch consumption and academic grades. Few studies have explored the relationship between a regular meal pattern throughout the day and academic grades. Many previous studies showed the positive effects of regular breakfast consumption on students' academic grades, and most of them focused on young students. $^{2} 741$ A study reported that a regular intake of breakfast and lunch was more crucial in grades 5 and 8 students compared with grade 11, whereas a regular intake of dinner was more likely related to academic achievement in grade 11 students compared with grade 5 and grade 8 students. ${ }^{42}$ In this study, eating breakfast, lunch and dinner five times or less per week accounted for $16.8 \%, 3.8 \%$ and $20.9 \%$, respectively. The frequency of eating dinner five times or less per week accounted for the highest proportion, possibly because the heavy schoolwork resulting in insufficient time for dinner or skipping dinner was served as a weight control measure among high school students. However, Chinese high school students were required to attend evening classes lasting for several hours, ${ }^{43}$ and skipping dinner may affect the learning efficiency of evening classes, which may have an important effect on students' academic grades. This 







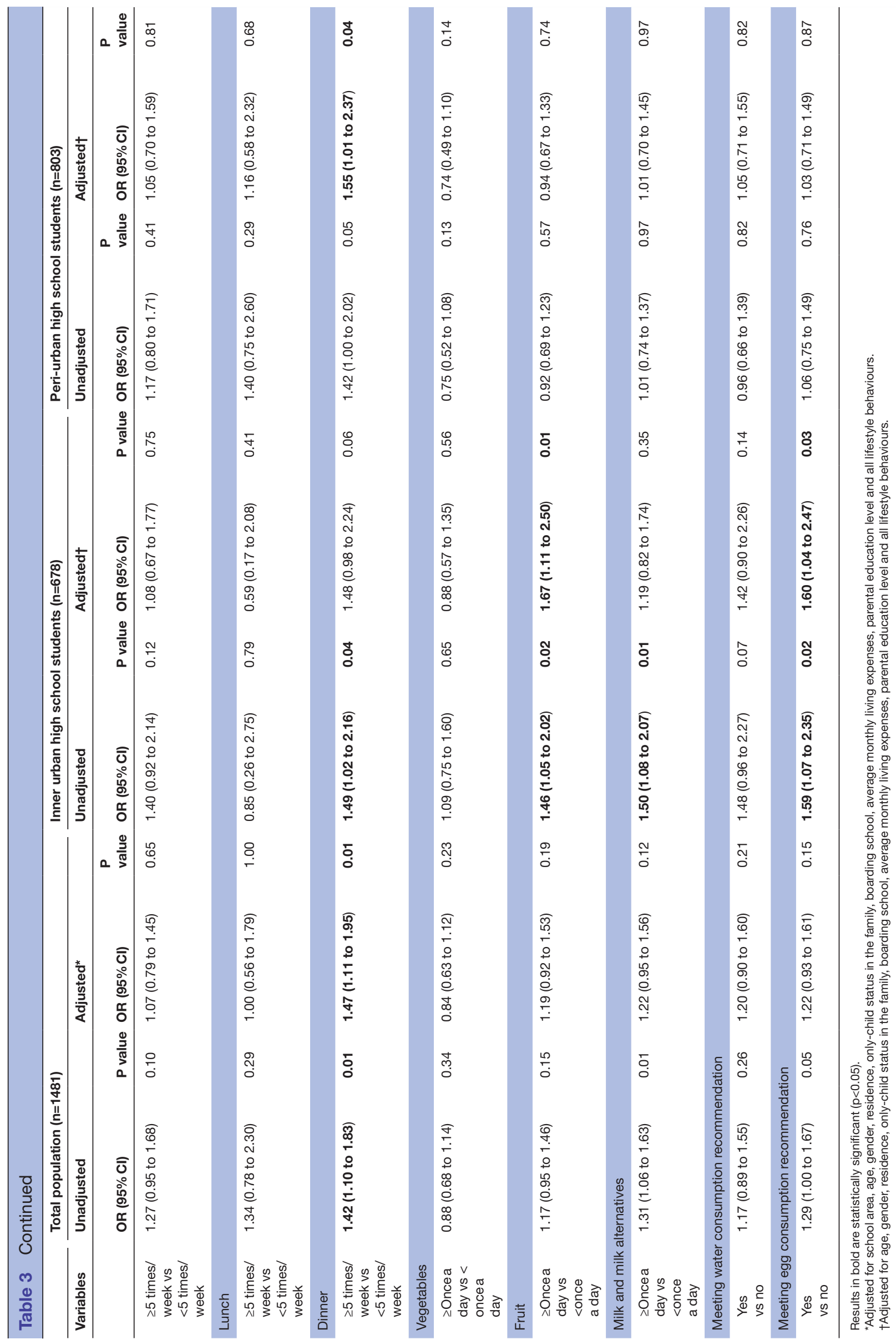


could be the reason that eating dinner regularly is positively correlated with academic grades. Health education related to regular meal patterns throughout the day must be conducted among high school students, particularly emphasising the importance of eating dinner. In IUHSSs and PUHSSs, high frequency of SSBs consumption was unlikely to obtain high academic grades. Additionally, among IUHSSs, meeting the recommendations for egg consumption and high frequency of fruit consumption had significant associations with high academic grades. These results were largely similar to previous studies, ${ }^{9} 2344$ which demonstrated that healthy dietary behaviours, such as frequent fruit consumption, were associated with high academic performance, whereas unhealthy dietary behaviours, including high frequency of SSBs consumption, were associated with poor academic performance.

In this study, no association was found between physical activity before or after school or on weekends, physical activity at school and academic grades in IUHSSs and PUHSSs. Most laboratory trials, cohort-based or crosssectional studies have demonstrated the positive effect of physical activity on cognitive function, ${ }^{45-48}$ while, studies on physical activity and academic grades have produced mixed results. ${ }^{40}$ A previous study found an inverse U-shaped curvilinear association between physical activity and academic achievement, thereby indicating that too little physical activity might impair the positive effects of physical activity on students' cognitive function and top sports students may have additional extracurricular physical activity that displaced the time spent on academics. ${ }^{51}$ Moreover, under the social, cultural and educational background of China, many schools have reduced the physical education curriculum to maximise the study time on important subjects for exams. ${ }^{18}$ This phenomenon is particularly prominent in high schools, as high school students need to prepare for the National College Entrance Examination. ${ }^{1}$ The results of this study showed that only $5.6 \%$ of the students before or after school or on weekends, and $19.5 \%$ of the students at school have more than 1 hour of physical activity. Considering these factors, the cut-off values of the recommendation for physical activity may be inappropriate to assess the association between physical activity and academic grades of high school students, and this could be the reason for the lack of significant association between physical activity and academic grades in IUHSSs and PUHSSs. This was also mentioned in a previous study targeting the association between physical activity and academic achievement among elementary school students. ${ }^{23}$ In addition, the questionnaire used for assessing the level of physical activity was not evaluated for its reliability and validity, the limited information provided by the questionnaire could be another reason for the lack of association. Choosing the appropriate method to assess the physical activity of high school students still needs to be explored in future studies, thereby to better explore its association with academic performance.

The findings demonstrated that the combined associations of multiple lifestyle behaviours resulted in a 
substantially higher likelihood of obtaining academic grades than the independent associations among IUHSSs, which was similar to the results of previous studies that investigated the combined effects of meeting recommendations for diet, physical activity, screen time and sleep on academic achievement of primary school students. ${ }^{22} 23$ Overall, one possible explanation for the positive association between multiple lifestyle behaviours and academic grades is that high school students with good academic grades usually have better self-control, which could contribute to high adherence to a healthy lifestyle. This study complements and extends these important findings in high school students. Given the importance of academic grades for high school students, this study could provide valuable references for health promotion initiatives that lead to improvement in academic grades. Interventions aiming to improve multiple lifestyle behaviours may have a greater positive effect on the academic grades of high school students than those focusing on a single lifestyle behaviour.

Regarding the potential mechanisms on the associations between lifestyle behaviours and academic grades, a previous study showed that adolescence is a critical period in which there is considerable reorganisation and growth of many brain structures, including the hippocampus related to learning and memory. ${ }^{52}$ Increasingly evidence demonstrates that the hippocampus is particularly responsive to the lifestyle influences during adolescence, previous studies showed that unhealthy diet and decreased exercise levels have been associated with decreased hippocampal neurogenesis and cognitive performance in rodent models. ${ }^{53-55}$ And the brainderived neurotrophic factor system appears to be a major mechanism underlying the effects of exercise and diet on neurogenesis and cognitive function. ${ }^{5256}$

This study has certain limitations. First, the use of crosssectional survey data reduces the researchers' ability to make direct causal inferences. Future longitudinal studies should be conducted to confirm the findings of this study. Second, lifestyle behaviours and academic grades were obtained by self-report that may introduce bias caused by self-enhancement and measurement flaws. Third, academic grades in this study is a comprehensive ranking of all compulsory subjects, however, students' lifestyle behaviours could have different influences on different subjects, ${ }^{23}$ the independent and combined associations between multiple lifestyle behaviours and academic grades among Chinese high school students could be explored in further studies according to the classification of students (science or liberal students), as well as the classification of subjects (Chinese, English, mathematics, physics, chemistry, biology, geography, history or politics). Fourth, being an exploratory study, questions on physical activity, sleep duration and screen time were adapted from the China Health and Nutrition Survey, ${ }^{57}$ and questions on dietary behaviours were adapted from Chinese Dietary Guideline 2016. ${ }^{34}$ Additionally, expert review and a pilot study were done before implementation. However, the questionnaire was not evaluated for its reliability and validity, and the unvalidated measures in the questionnaire designed to measure the lifestyle behaviours and academic grades may raise potential issues related to reliability. Further studies should consider using a verified questionnaire or validating the questionnaire before administration. Fifth, the participants who were investigated in this study could only reflect the situation of high school students in Chongqing to a certain extent, and cannot be generalisable to all high school students across China. Further nationally representative studies are warranted.

\section{CONCLUSIONS}

This study demonstrated that there is a correlation between the lifestyle behaviours of high school students and academic grades, and cumulative associations between multiple healthy lifestyle behaviours and academic grades appear to be stronger than the independent associations. These findings are particularly applicable to IUHSSs. The importance of multiple healthy lifestyle behaviours for the academic grades of high school students should be considered by public health decision-makers and school principals or management bodies in the high school settings.

\section{Author affiliations}

${ }^{1}$ School of Public Health and Management, Chongqing Medical University, Chongqing, China

${ }^{2}$ West China School of Public Health and West China Fourth Hospital, Sichuan University, Chengdu, China

${ }^{3}$ Research Center for Medicine and Social Development, Chongqing Medical University, Chongqing, China

${ }^{4}$ The Innovation Center for Social Risk Governance in Health, Chongqing Medical University, Chongqing, China

${ }^{5}$ Department of Clinical Nutrition, West China Hospital, Sichuan University, Chengdu, China

${ }^{6}$ California University of Science and Medicine, Colton, California, USA

${ }^{7}$ The Second Clinical College, Chongqing Medical University, Chongqing, China ${ }^{8}$ Environmental \& Occupational Health, School of Public Health, University of Nevada, Las Vegas, Nevada, USA

${ }^{9}$ Chongqing Key Laboratory of Child Nutrition and Health, Children's Hospital of Chongqing Medical University, Chongqing, China

Acknowledgements We would like to thank the support of the Postgraduate Scientific Research and Innovation Project of Chongqing (CYS19208). Also, we would like to thank all the teachers and class leaders who helped us coordinate the survey. We thank all the participants for their support of this project. We also would like to acknowledge all the investigators who participated in the survey.

Contributors ZC carried out investigation, data analysis and wrote the paper. JX, $\mathrm{HZ}$ and HP provided help with the investigation and data collection. JX, CA, ZZ and MS provided the assistance in reviewing the paper. YZ provided guidance in study design, organised the investigation, and is the corresponding author. And $Y Z$ acted as guarantor for the overall content of the article. All authors approved the final manuscript.

Funding This study was funded by the Postgraduate Scientific Research and Innovation Project of Chongqing, grant number CYS19208.

Competing interests None declared.

Patient consent for publication Not applicable.

Ethics approval This study was reviewed and approved by the Ethics Committee of Chongqing Medical University (record number 2016001). Informed consent was 
obtained from all participants, and they could voluntarily participate or withdraw from the study at any stage.

Provenance and peer review Not commissioned; externally peer reviewed.

Data availability statement Data are available on reasonable request. The datasets used and/or analyzed during the current study are available from the corresponding author on reasonable request.

Supplemental material This content has been supplied by the author(s). It has not been vetted by BMJ Publishing Group Limited (BMJ) and may not have been peer-reviewed. Any opinions or recommendations discussed are solely those of the author(s) and are not endorsed by BMJ. BMJ disclaims all liability and responsibility arising from any reliance placed on the content. Where the content includes any translated material, BMJ does not warrant the accuracy and reliability of the translations (including but not limited to local regulations, clinical guidelines, terminology, drug names and drug dosages), and is not responsible for any error and/or omissions arising from translation and adaptation or otherwise.

Open access This is an open access article distributed in accordance with the Creative Commons Attribution Non Commercial (CC BY-NC 4.0) license, which permits others to distribute, remix, adapt, build upon this work non-commercially, and license their derivative works on different terms, provided the original work is properly cited, appropriate credit is given, any changes made indicated, and the use is non-commercial. See: http://creativecommons.org/licenses/by-nc/4.0/.

\section{ORCID iDs}

Zhengjie Cai http://orcid.org/0000-0002-5955-6949

Jinli Xian http://orcid.org/0000-0003-1100-9898

Yong Zhao http://orcid.org/0000-0001-9290-109X

\section{REFERENCES}

1 Kirkpatrick R, Zang Y. The negative influences of Exam-Oriented education on Chinese high school students: Backwash from classroom to child. Language Testing in Asia 2011;1:36.

2 Yao J, Liu Y, Zhou S. Effect of eating breakfast on cognitive development of elementary and middle school students: an empirica study using large-scale provincial survey data. Med Sci Monit 2019;25:8843-53.

3 Chen W-L, Chen J-H. Consequences of inadequate sleep during the College years: sleep deprivation, grade point average, and College graduation. Prev Med 2019;124:23-8.

4 Stea TH, Torstveit MK. Association of lifestyle habits and academic achievement in Norwegian adolescents: a cross-sectional study. BMC Public Health 2014;14:829.

5 Scott SP, De Souza MJ, Koehler K, et al. Combined iron deficiency and low aerobic fitness doubly burden academic performance among women attending university. J Nutr 2017;147:104-9.

6 Burrows T, Goldman S, Pursey K, et al. Is there an association between dietary intake and academic achievement: a systematic review. J Hum Nutr Diet 2017;30:117-40.

7 Ptomey LT, Steger FL, Schubert MM, et al. Breakfast intake and composition is associated with superior academic achievement in elementary schoolchildren. J Am Coll Nutr 2016;35:326-33.

8 Kooshki A, Mohammadi M, Rivandi M. Nutritional intake and its association with educational achievement in high-school students in Islamic Republic of Iran. East Mediterr Health J 2018;24:532-7.

9 Burrows T, Goldman S, Olson RK, et al. Associations between selected dietary behaviours and academic achievement: a study of Australian school aged children. Appetite 2017;116:372-80.

10 Hötting K, Röder B. Beneficial effects of physical exercise on neuroplasticity and cognition. Neurosci Biobehav Rev 2013;37:2243-57.

11 Edwards JU, Mauch L, Winkelman MR. Relationship of nutrition and physical activity behaviors and fitness measures to academic performance for sixth graders in a Midwest City school district. J Sch Health 2011;81:65-73.

12 Resaland GK, Aadland E, Moe VF, et al. Effects of physical activity on schoolchildren's academic performance: the active smarter kids (ASK) cluster-randomized controlled trial. Prev Med 2016;91:322-8.

13 Singh AS, Saliasi E, van den Berg V, et al. Effects of physical activity interventions on cognitive and academic performance in children and adolescents: a novel combination of a systematic review and recommendations from an expert panel. Br J Sports Med 2019;53:640-7.

14 Hysing M, Harvey AG, Linton SJ, et al. Sleep and academic performance in later adolescence: results from a large populationbased study. J Sleep Res 2016;25:318-24.
15 Wolfson AR, Carskadon MA. Understanding adolescents' sleep patterns and school performance: a critical appraisal. Sleep Med Rev 2003;7:491-506.

16 Adelantado-Renau M, Moliner-Urdiales D, Cavero-Redondo I, et al. Association between screen media use and academic performance among children and adolescents: a systematic review and metaanalysis. JAMA Pediatr 2019;173:1058-1067.

17 Yan $\mathrm{H}$, Zhang R, Oniffrey $\mathrm{T}$, et al. Associations among screen time and unhealthy behaviors, academic performance, and well-being in Chinese adolescents. Int J Environ Res Public Health 2017;14:596.

18 Zhang Y, Zhang D, Jiang Y, et al. Association between physical activity and teacher-reported academic performance among fifth-graders in Shanghai: a quantile regression. PLoS One 2015;10:e0115483.

19 Martínez-Gómez D, Veiga OL, Gómez-Martínez S, et al. GenderSpecific influence of health behaviors on academic performance in Spanish adolescents: the AFINOS study. Nutr Hosp 2012;27:724-30.

20 Bradley BJ, Greene AC. Do health and education agencies in the United States share responsibility for academic achievement and health? A review of 25 years of evidence about the relationship of adolescents' academic achievement and health behaviors. J Adolesc Health 2013;52:523-32.

21 Kamath CC, Vickers KS, Ehrlich A, et al. Clinical review: behavioral interventions to prevent childhood obesity: a systematic review and metaanalyses of randomized trials. J Clin Endocrinol Metab 2008;93:4606-15.

22 Ickovics JR, Carroll-Scott A, Peters SM, et al. Health and academic achievement: cumulative effects of health assets on standardized test scores among urban youth in the United States. J Sch Health 2014;84:40-8.

23 Faught EL, Ekwaru JP, Gleddie D, et al. The combined impact of diet, physical activity, sleep and screen time on academic achievement: a prospective study of elementary school students in nova Scotia, Canada. Int J Behav Nutr Phys Act 2017;14:29.

24 Chortatos A, Terragni L, Henjum S, et al. Consumption habits of school canteen and non-canteen users among Norwegian young adolescents: a mixed method analysis. BMC Pediatr 2018;18:328-28.

25 Ragelienè T, Grønhøj A. The influence of Peers' and siblings' on children's and adolescents' healthy eating behavior. A systematic literature review. Appetite 2020;148:104592.

$26 \mathrm{Li} \mathrm{D.} \mathrm{The} \mathrm{study} \mathrm{about} \mathrm{the} \mathrm{grade} \mathrm{and} \mathrm{psychological} \mathrm{health} \mathrm{of} \mathrm{the}$ boarders and day students-For example Shandong experimental high school. Shandong Normal University 2007.

27 Pan XD, Guan NN. Analysis of the relationship between mental health status and academic performance of medical students. Medical Education Research and Practice 2019;27:68-72.

28 Haddad MR, Sarti FM. Sociodemographic determinants of health behaviors among Brazilian adolescents: trends in physical activity and food consumption, 2009-2015. Appetite 2020;144:104454.

29 Zhao P. Too complex to be managed? new trends in periurbanisation and its planning in Beijing. Cities 2013;30:68-76.

30 Run J, Sun Q. The spatio-temporal evolution of the County economic disparity in Chongqing City since its Municipality. Journal of Southwest University 2020;42:106-16.

31 Shrewsbury VA, Foley BC, Flood VM, et al. School-Level socioeconomic status influences adolescents' health-related lifestyle behaviors and intentions. J Sch Health 2018;88:583-9.

32 Yan $\mathrm{H}$, Zhang R, Oniffrey TM, et al. Associations among screen time and unhealthy behaviors, academic performance, and well-being in Chinese adolescents. Int J Environ Res Public Health 2017;14:596.

33 National Children's Medical Center, Shanghai Chidren's Medical Center, Shanghai Institute of Physical Education. Clinical guidelines development and evaluation center of children's hospital of Fudan University. Physical activity guidelines for chinese children and adolescents. Shanghai, China, 2018. Available: http://www.shanghai. gov.cn/nw2/nw2314/nw2315/nw17239/nw17240/u21aw1286214. html

34 Chinese Nutrition Society. Dietary guidelines for chinese school-age children 2016. Beijing, China: People's Medical Publishing House, 2016. http://dg.cnsoc.org/article/04/8a2389fd575f695101577a54 $5 \mathrm{~b} 0102 \mathrm{db} . \mathrm{htm}$

35 Azeredo CM, de Rezende LFM, Canella DS, et al. Dietary intake of Brazilian adolescents. Public Health Nutr 2015;18:1215-24.

36 Guthold R, Stevens GA, Riley LM, et al. Global trends in insufficient physical activity among adolescents: a pooled analysis of 298 population-based surveys with 1.6 million participants. Lancet Child Adolesc Health 2020;4:23-35.

37 Chen $\mathrm{X}$, Wang Y. Is ideal body image related to obesity and lifestyle behaviours in African American adolescents? Child Care Health Dev 2012;38:219-28. 
38 Lim J, Dinges DF. A meta-analysis of the impact of short-term sleep deprivation on cognitive variables. Psychol Bull 2010;136:375-89.

39 Rasch B, Born J. About sleep's role in memory. Physiol Rev 2013;93:681-766

40 Wang ZQ, Bai X. An empirical study on the regional differences in the development level of compulsory education in the Province: a case of Chongqing, a pilot area for coordinating urban and rural education development. Modern Education Management 2020:63-9.

41 Littlecott HJ, Moore GF, Moore L, et al. Association between breakfast consumption and educational outcomes in 9-11-year-old children. Public Health Nutr 2016;19:1575-82.

$42 \mathrm{Kim} \mathrm{H-YP,} \mathrm{Frongillo} \mathrm{EA,} \mathrm{Han} \mathrm{S-S,} \mathrm{et} \mathrm{al.} \mathrm{Academic} \mathrm{performance} \mathrm{of}$ Korean children is associated with dietary behaviours and physical status. Asia Pac J Clin Nutr 2003;12:186-92.

43 Liu X, Zhao Z, Jia C, et al. Sleep patterns and problems among Chinese adolescents. Pediatrics 2008;121:1165-73.

44 Dumuid D, Olds T, Martín-Fernández J-A, et al. Academic performance and lifestyle behaviors in Australian school children: a cluster analysis. Health Educ Behav 2017;44:918-27.

45 Donnelly JE, Hillman CH, Castelli D, et al. Physical activity, fitness, cognitive function, and academic achievement in children: a systematic review. Med Sci Sports Exerc 2016;48:1223-4.

46 Krafft CE, Schaeffer DJ, Schwarz NF, et al. Improved frontoparietal white matter integrity in overweight children is associated with attendance at an after-school exercise program. Dev Neurosci 2014;36:1-9.

47 Chaddock L, Hillman CH, Pontifex MB, et al. Childhood aerobic fitness predicts cognitive performance one year later. J Sports Sci 2012;30:421-30.
48 Walsh JJ, Barnes JD, Cameron JD, et al. Associations between 24 hour movement behaviours and global cognition in US children: a cross-sectional observational study. Lancet Child Adolesc Health 2018;2:783-91.

49 Hollar D, Messiah SE, Lopez-Mitnik G, et al. Effect of a two-year obesity prevention intervention on percentile changes in body mass index and academic performance in low-income elementary school children. Am J Public Health 2010;100:646-53.

50 Donnelly JE, Hillman CH, Greene JL, et al. Physical activity and academic achievement across the curriculum: results from a 3-year cluster-randomized trial. Prev Med 2017;99:140-5.

51 Syväoja HJ, Kantomaa MT, Ahonen T, et al. Physical activity, sedentary behavior, and academic performance in Finnish children. Med Sci Sports Exerc 2013;45:2098-104.

52 Hueston CM, Cryan JF, Nolan YM. Stress and adolescent hippocampal neurogenesis: diet and exercise as cognitive modulators. Transl Psychiatry 2017;7:e1081.

53 Opendak M, Gould E. Adult neurogenesis: a substrate for experience-dependent change. Trends Cogn Sci 2015;19:151-61.

54 Boitard C, Etchamendy N, Sauvant J, et al. Juvenile, but not adult exposure to high-fat diet impairs relational memory and hippocampa neurogenesis in mice. Hippocampus 2012;22:2095-100.

55 van Praag $\mathrm{H}$. Neurogenesis and exercise: past and future directions. Neuromolecular Med 2008;10:128-40.

56 Bekinschtein P, Oomen CA, Saksida LM, et al. Effects of environmental enrichment and voluntary exercise on neurogenesis, learning and memory, and pattern separation: BDNF as a critical variable? Semin Cell Dev Biol 2011;22:536-42.

57 CHNS. China health and nutrition survey, 2020. Available: https:// www.cpc.unc.edu/projects/china 